cardiopulmonary bypass, or an off-pump hybrid procedure. As there was no need to exchange the well-functioning mitral prosthesis itself, we decided to go for the last option.

Approaching the paravalvular leak from the left ventricular apex turned out to be much easier than expected. The guidewire, which was approached first, fell more or less by itself into the dehiscence between the sewing ring and annulus. However, there was one delicate situation. During the pushing of the Amplatzer device out of the sheath, one leaflet of the mitral valve was blocked. It was hard to determine whether the umbrella itself or the sheath was responsible was responsible for this blockade. We pulled the introducer sheath back without detaching the umbrella. Fortunately, valve function returned to normal, proving that the sheath did indeed compromise function.

We believe that in this particular situation, a hybrid procedure, reduced the risk of the operative procedure significantly.

\section{References}

1. Schmitz C, Esmailzadeh B, Herberg U, Lang N, Sodian R, Kozlik-Feldmann R, et al. Hybrid procedures can reduce the risk of congenital cardiovascular surgery. Eur J Cardiothorac Surg. 2008;34:718-25.

2. Sivakumar K, Shahani J. Transcatheter closure of paravalvular mitral prosthetic leak with resultant hemolysis. Int J Cardiol. 2007;115:e39-40.

3. Webb JG, Pate GE, Munt BI. Percutaneous closure of an aortic prosthetic paravalvular leak with an Amplatzer duct occluder. Catheter Cardiovasc Interv. 2005;65: 69-72.

\title{
Complete lung parenchyma-sparing resection of the right main stem bronchus and bronchus intermedius
}

\author{
Justin H. Booth, BS, ${ }^{\mathrm{a}, \mathrm{c}}$ Roosevelt Bryant III, MD, ${ }^{\mathrm{b}, \mathrm{c}}$ Ara Vaporciyan, MD, ${ }^{\mathrm{b}}$ and David L. S. Morales, MD, ${ }^{\mathrm{b}, \mathrm{c}}$ \\ Houston, Tex
}

Pulmonary carcinoid tumors account for only $1 \%$ to $2 \%$ of all lung cancers, but they are the most common primary pulmonary neoplasm in the pediatric population. ${ }^{1}$ They are classified as typical or atypical on the basis of their clinical behavior and histologic findings. ${ }^{2}$ Although both types are considered malignant, the much more common typical carcinoids rarely metastasize to regional lymph nodes or distally and have an excellent prognosis with a 10-year survival of $82 \%$ to $100 \% .^{2}$ Complete surgical resection is the treatment of choice. Inasmuch as only negative margins are necessary and radical resection is rarely needed, every effort should be made to preserve as much lung parenchyma as possible.

We present a case of a typical carcinoid of the right airway treated with resection of the right main stem bronchus (RMB) and bronchus intermedius (RBI) with carinal reconstruction using the right lower lobe (RLL) and right middle lobe (RML) carina and creation of a pig bronchus (bronchus suis).

From the University of Texas Medical School at Houston, ${ }^{a}$ the Michael E. Debakey Department of Surgery, Baylor College of Medicine, ${ }^{\mathrm{b}}$ and the Division of Congenital Heart Surgery, Texas Children's Hospital, ${ }^{\mathrm{c}}$ Houston, Tex.

Received for publication April 1, 2008; revisions received April 23, 2008; accepted for publication May 13, 2008; available ahead of print March 9, 2009.

Address for reprints: Roosevelt Bryant III, MD, Texas Children's Hospital, 6621 Fannin St, Houston, TX 77030 (E-mail: rxbryan1@texaschildrenshospital.org).

J Thorac Cardiovasc Surg 2010;139:222-4

$0022-5223 / \$ 36.00$

Copyright (c) 2010 by The American Association for Thoracic Surgery

doi:10.1016/j.jtcvs.2008.05.070

\section{CLINICAL SUMMARY}

An 11-year-old boy with a history of upper respiratory infections and chronic fatigue was admitted with hemoptysis. Computed tomography of the chest and examination with a rigid bronchoscope demonstrated a right bronchial mass almost completely obliterating both the RMB and RBI. Biopsy demonstrated a grade I neuroendocrine carcinoid.

After intubation with a micro-long endotracheal tube placed in the left main stem bronchus, a right posterolateral thoracotomy incision was made. A vascular pedicled intercostal muscle was harvested.

Anteromedial retraction of the lung allowed visualization of the posterior aspect of the RMB. The tumor extended from the RMB orifice to halfway down the RBI along the airway's medial wall and did not seem to involve the right upper lobe (RUL) bronchus. Although the mass did abut the esophagus and pulmonary artery, there was no adherence. After resection of the level 10 and 11 lymph nodes, development of the minor fissure, and circumferential dissection of the RMB and RBI as well as all the hilar vessels, the RUL bronchus was transected. The RBI was transected at the level of the RML and RLL orifices and the RMB was transected at the carina. All tissues attached to the tumor and the level 7 lymph nodes were excised en bloc with the mass (Figure 1, A). Frozen sections of the bronchial and carinal margins were free of malignancy.

A complete hilar release was performed with the inferior vena cava released down to the diaphragm. The trachea 

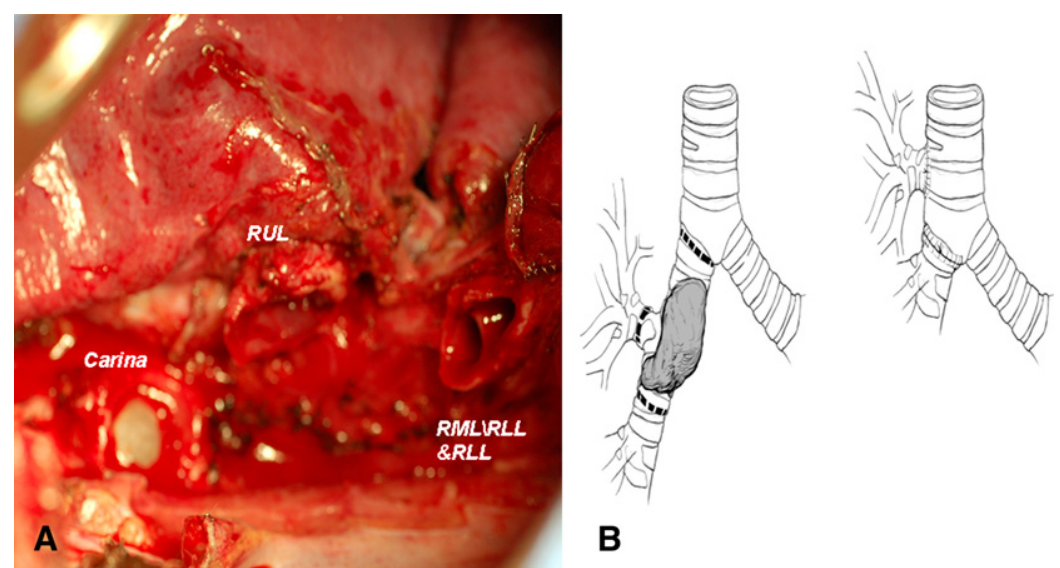

FIGURE 1. A, Surgical site after excision of tumor. $R U L$, Right upper lobe; $R M L \backslash R L L$, right middle lobe/right lower lobe. B, Schematic of reconstruction: On the left, the dashed lines indicate the margins of tumor resection. On the right is shown the carinal reconstruction with right middle and lower lobes and creation of a bronchus suis.

was mobilized anteriorly and posteriorly. The carina was reconstructed by an interrupted anastomosis to the RML/ RLL carina. The RUL was reimplanted into the cartilaginous section of the anterolateral trachea 3 rings above the carina. (Figure 1,B) A flexible bronchoscope was used to ensure airway patency and to clear secretions. The anastomoses were buttressed with the previously harvested intercostal pedicle flap, with care taken not to completely encircle any of the airways. The patient was extubated the night of the operation and was discharged home on postoperative day 7 , having had no complications. Pathologic examination revealed a $3-\mathrm{cm}$ grade I neuroendocrine carcinoma with extension through the bronchial wall. All lymph nodes and margins were negative for cancer. No additional therapy was given. Twenty months later, the patient is disease free and asymptomatic with strenuous exercise. His follow-up bronchoscopic study and computed tomographic scan are shown (Figure 2, $A$ and $B$ ).

\section{DISCUSSION}

Bronchopulmonary carcinoid tumors are usually well localized and, therefore, complete resection is usually the only treatment modality necessary. Surgeons have always tried to minimize their resection area with this tumor because of its indolent oncologic behavior, trying to salvage as much lung parenchyma as possible. This has led to a more frequent use of bronchoplastic resections such as sleeve resections and bronchotomy and primary anastomosis.,

This case presents a unique opportunity in attempting to salvage the lung parenchyma inasmuch as the tumor involved much of the RMB and RBI, but spared the RUL bronchus. Therefore, an extensive resection of the entire RMB and RBI with reimplantation of the RUL into the
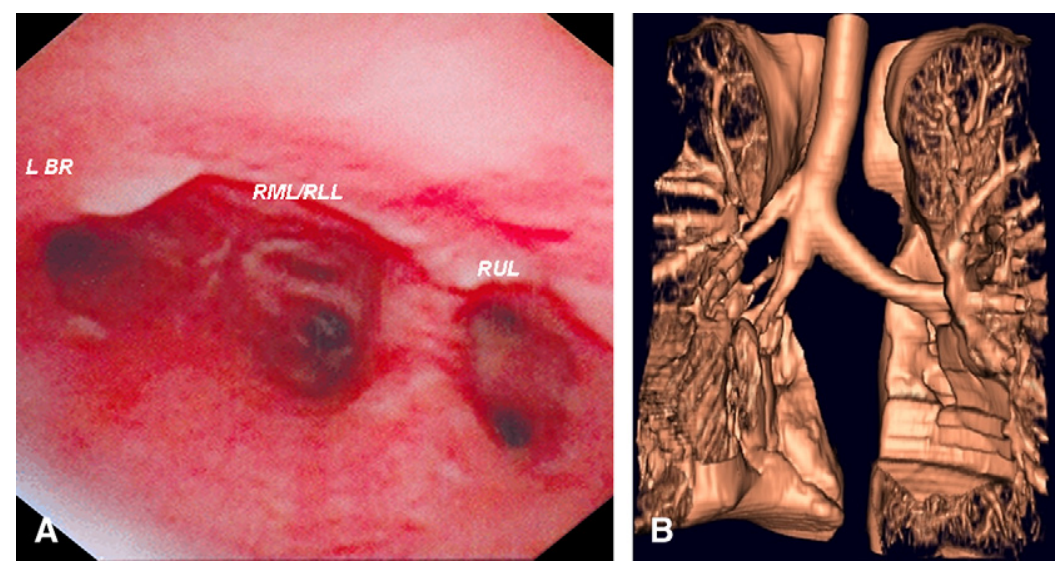

FIGURE 2. A, Bronchoscopic examination at 6 months. $L B R$, Left main stem bronchus; $R M L / R L$, right middle lobe/right lower lobe; $R U L$, right upper lobe. $\mathrm{B}$, Three-dimensional computed tomographic reconstruction of airways 6 months postoperatively. 
trachea, creating a pig bronchus (bronchus suis), was accomplished. Full mobilization of the hilar vessels and an aggressive hilar release allowed this to be done without transection of any vessels. Carinal reconstruction was followed by anastomosing the secondary carina, which had a similar circumference to the RMB, directly to the carina. This technique allowed complete preservation of lung parenchyma. In the years before this diagnosis, the patient was thought to have a sedentary personality because he tired easily and did not participate in athletic activities. A year after resection, this eighth grader with a widely patent and nonobstructed airway is running varsity cross-country. We hope that his com- plete parenchyma-sparing airway resection contributed to his excellent functional outcome.

\section{References}

1. Andrassy RJ, Feldtman RW, Stanford W. Bronchial carcinoid tumors in children and adolescents. J Pediatr Surg. 1977;12:513-7.

2. Ferguson MK, Landreneau RJ, Hazelrigg SR, Altorki NK, Naunheim KS, Zwischenberger JB, et al. Long-term outcome after resection for bronchial carcinoid tumors. Eur J Cardiothorac Surg. 2000;18:156-61.

3. Filosso PL, Rena O, Donati G, Casadio C, Ruffini E, Papalia E, et al. Bronchial carcinoid tumors: surgical management and long-term outcome. J Thorac Cardiovasc Surg. 2002; 123:303-9.

4. McMullan DM, Wood DE. Pulmonary carcinoid tumors. Semin Thorac Cardiovasc Surg. 2003;15:289-300.

\title{
Use of unidirectional endobronchial valves for the treatment of giant emphysematous bulla
}

\author{
Mario Santini, MD, Alfonso Fiorello, MD, Vincenzo Giuseppe Di Crescenzo, MD, \\ Giovanni Vicidomini, MD, PhD, Luigi Busiello, MD, and Paolo Laperuta, MD, Naples, Italy
}

We report the successful treatment of a giant emphysematous bulla (GEB) by bronchoscopic placement of unidirectional endobronchial valve (EBV; Zephyr; Emphasys Medical, Inc, Redwood City, Calif) in a patient not able to undergo open thoracotomy.

\section{CLINICAL SUMMARY}

A 68-year-old man who had a history of emphysema and recurrent left pneumothorax treated by pleurodesis with video-assisted thoracoscopic surgery 7 months previously was admitted at our hospital because of dyspnea. All laboratory examinations returned normal results. Blood gas values with room air were as follows: $\mathrm{pH} 7.4, \mathrm{PCO}_{2} 36.2 \mathrm{~mm} \mathrm{Hg}$, and $\mathrm{Po}_{2} 64.9 \mathrm{~mm} \mathrm{Hg}$. The forced vital capacity was 1.35 $\mathrm{L}$ ( $49 \%$ of predicted) with a forced expiratory volume in 1 second of $1.2 \mathrm{~L}$ ( $51 \%$ of predicted).

Computed tomography showed multiple bullae, with a single, larger bulla occupying almost the entire left upper hemithorax and causing pulmonary compression and a mediastinal shift to right (Figure 1, $A$ ).

From the Thoracic Surgery Unit, Second University of Naples, Naples, Italy. Received for publication April 14, 2008; accepted for publication May 5, 2008; available ahead of print Feb 5, 2009.

Address for reprints: Mario Santini, MD, Chirurgia Toracica-Seconda Università di Napoli, Piazza Miraglia, 2, I-80138 Naples, Italy (E-mail: mario.santini@unina2.it). J Thorac Cardiovasc Surg 2010;139:224-6

$0022-5223 / \$ 36.00$

Copyright (C) 2010 by The American Association for Thoracic Surgery doi:10.1016/j.jtcvs.2008.05.069
Previous pleurodesis and poor respiratory condition of the patient discouraged us from performing bullectomy, and the patient was reviewed for endobronchial treatment. We hypothesized that EBVs deflating the bulla might promote the re-lexpansion of the compressed adjacent lungs and improve respiratory function. After informed consent had been obtained, the patient was brought to operating room. A general anesthetic was used to ensure good control of ventilation and cough during the procedure. Anesthesia was induced, and the patient was intubated with an endotracheal tube (size 9.0) to reduce the need for high airway pressures during bronchoscopy and manual ventilation. Flexible bronchoscopy was performed, and examination of the segmental airway anatomy showed compression of the left lower bronchus. According to the instructions supplied with the valve, ${ }^{1}$ two Zephyr 4.0 EBVs were sequentially positioned into the superior trunk bronchus (culmen) of the left upper lobe and into the lingular bronchus (Figure 2, A). Subjective improvement of dyspnea, with diminution in size of the bulla and centralization of mediastinum visible on chest radiography, were noted just the next day. The patient was discharged on postoperative day 5 .

As of 1 month after the procedure, the patient was doing well without recurrence of the pneumothorax. Respiratory function test results showed a forced vital capacity of $1.85 \mathrm{~L}$ ( $67 \%$ of predicted) and a forced expiratory volume in 1 second of $1.7 \mathrm{~L}$ ( $70 \%$ of predicted). The valves have remained in place, and no attempts at removal have been made. Computed tomography showed an almost complete disappearance of the bulla, with re-expansion of the neighboring lung (Figure 1,B). 